Elisabeth Meloni Vieira 1

Wilza Vieira Villela ${ }^{2}$

Marina Ferreira Réa 2

Maria Eugênia Lemos Fernandes 3

Emília de Franco 3

Giane Ribeiro 3

\section{Alguns aspectos do comportamento sexual e prática de sexo seguro em homens do Município de São Paulo}

\author{
Key aspects of sexual behavior and safer sex \\ practice among men from the city of São Paulo
}

1 Hospital das Clínicas, Departamento de Medicina Social, Faculdade de Medicina de Ribeirão Preto, Universidade de São Paulo. Av. dos Bandeirantes 3900 , 2 o andar, Ribeirão Preto, $S P$ 14049-900, Brasil.

2 Instituto de Saúde, Secretaria de Estado da Saúde de São Paulo. Rua Santo Antônio 590, 2 o andar, São Paulo, SP 01314-000, Brasil.

3 Associação Saúde da Família. Rua Francisco Iasi 94, São Paulo, SP 05407-050, Brasil.

\begin{abstract}
Heterosexual relations currently constitute the predominant route of HIV transmission to women in Brazil. Few studies have approached male sexual behavior taking women into account. This study included 597 men ages 21 to 50 in São Paulo city and explored several as pects pertaining to safer sex, such as sexual partners, knowledge of sexually transmitted diseases (STDS)/AIDS, risk perception, and protective practices aimed at AIDS prevention. The results showed that, similar to other countries, the proportion of men who have sex with men is low (5\%), that younger men adopt more protective behavior than older men regarding multiple partners and condom use, that knowledge of AIDS is widespread (but that there is still a lack of knowledge concerning STDs in general), and that although risk perception increases protective behavior, presumption of the partner's behavior influences this perception, placing men and women in a vulnerable situation.
\end{abstract}

Key words Sexually Transmitted Diseases; Acquired Immunodeficiency Syndrome; Sexual Behavior; Men

Resumo A via heterossexual é atualmente o modo dominante de transmissão do HIV em mulheres no Brasil. Poucos estudos têm abordado o comportamento sexual masculino heterossexual. Um estudo realizado com 597 homens de 21 a 50 anos na cidade de São Paulo explorou vários aspectos relativos ao sexo mais seguro, tais como parceiras sexuais, conhecimento de Doenças Sexualmente Transmissíveis (DST)/AIDS, percepção de risco e práticas auto protetoras, visando assim contribuir para a prevenção da AIDS. Os resultados mostram que, à semelhança de outros países, a proporção de homens que se engajam em práticas sexuais com outros homens é pequena (5\%), que maior proporção de jovens têm um comportamento de auto proteção, seja em relação à múltiplas parcerias simultâneas ou ao uso do preservativo, que o conhecimento sobre a AIDS é muito difundido, mas sobre as DST é bem menor; que embora a percepção de risco aumente o comportamento de auto proteção, a presunção do comportamento da parceira influencia esta percepção de risco colocando em situação vulnerável homens e mulheres.

Palavras-chave Doenças Sexualmente Transmissíveis; Síndrome de Imunodeficiência Adquirida; Comportamento Sexual; Homens 


\section{Introdução}

A AIDS figura como segunda causa de morte em adultos (20 a 49 anos) no Brasil. A transmissão heterossexual foi responsável por quase metade dos casos $(49,7 \%)$ de AIDS notificados em 1998, se excluirmos os de transmissão ignorada. A via heterossexual é hoje o modo dominante da transmissão do Vírus da Imunodeficiência Humana (HIV) na população feminina (MS, 1998). Em decorrência da transmissão vertical do HIV, o aumento de AIDS em mulheres é acompanhado do crescimento do número de casos em crianças.

A prevenção do HIV é feita pelo controle do sangue e hemoderivados, uso de seringas e agulhas descartáveis e prática do sexo mais seguro. Esta última inclui: (a) usar preservativo em todas as relações sexuais(uso consistente), (b) praticar outras formas de sexo mais seguro, na qual não haja troca de fluidos orgânicos, como por exemplo, práticas sem penetração, (c) abster-se ou postergar relações sexuais e (d) reduzir o número de parceiros sexuais (Dalabetta et al., 1997). A prevenção também é feita através do controle das doenças sexualmente transmissíveis (DST). Em um estudo, realizado na Tanzânia, encontrou-se redução da ordem de $42 \%$ na incidência do HIV em uma comunidade, depois do controle e tratamento das DST (Grosskurth et al., 1995).

Segundo Giffin (1998), nas pesquisas voltadas para a compreensão dos determinantes de risco entre os diferentes grupos populacionais, não existe homogeneidade. Ou seja, enquanto os segmentos da população inicialmente mais afetados pela epidemia - homossexuais masculinos, profissionais do sexo têm sido exaustivamente pesquisados e monitorados em todo o mundo, estudos sobre mulheres existem em menor quantidade, tendo sido incrementados apenas a partir dos impactos crescentes da heterossexualização da epidemia. Embora haja menor número de estudos sobre mulheres e AIDS realizados em diferentes grupos populacionais femininos, utilizando abordagens metodológicas distintas, existe concordância com relação à dificuldade das mulheres negociarem práticas sexuais mais seguras, devido as diferenças de poder entre os gêneros, em particular, na esfera da sexualidade. Essas diferenças colocam as mulheres em desvantagem em relação à sua autoproteção (Goldstein, 1994; Guimarães, 1994; Heise \& Elias, 1995; Gomez \& Marin, 1996; Barbosa, 1997). Autores que estudam estes aspectos afirmam que os esforços de prevenção do HIV em mulheres, só terão sucesso efetivo na dependência da mudança de comportamento masculino com o aumento do uso do preservativo (Campbell, 1995).

Ainda existe desconhecimento em relação ao comportamento dos heterossexuais masculinos na prevenção do HIV, sendo necessário mais estudos sobre masculinidade, em particular no que se refere ao comportamento sexual e reprodutivo, e à vivência dos padrões de gênero dos homens (Villela, 1996, 1997); especialmente após a Conferência de População e Desenvolvimento do Cairo, realizada em 1994, quando os homens foram incluídos nas questões de saúde sexual e reprodutiva (Conferência Internacional sobre População e Desenvolvimento, 1994).

Visando uma abordagem inicial, foi realizado um estudo na cidade de São Paulo sobre o envolvimento masculino em saúde reprodutiva, que também abordou as estratégias de auto proteção frente às conseqüências não desejadas da relação sexual. Este artigo apresenta alguns resultados sobre parcerias sexuais, conhecimento de DST/AIDS, percepção de risco e prática de sexo seguro visando a prevenção do HIV/AIDS.

\section{Material e métodos}

O estudo incluiu a coleta de dados qualitativos e quantitativos. O componente quantitativo consistiu da aplicação de um questionário, por entrevistadoras treinadas, a 597 homens entre 21 e 50 anos, residentes no município de São Paulo, que estivessem trabalhando no período de outubro de 1996 a janeiro de 1997.

O questionário foi em parte elaborado a partir da análise preliminar de seis grupos focais masculinos e femininos realizados com 43 participantes no período de maio e junho de 1996, mas também foram utilizadas como fontes questionários de outras pesquisas, como o National Survey of Sexual Attitudes and Lifestyles (SCPR, 1990) e a Pesquisa Nacional de Demografia e Saúde (PNDS) - 1996 (BEMFAM/Macro International, 1997). Este instrumento foi pré-codificado e pré-testado e continha 66 perguntas organizadas em quatro seções: (1) dados sócio-demográficos, (2) saúde reprodutiva, (3) comportamento sexual e (4) papéis de gênero.

Em vista das limitações orçamentárias de se obter uma amostra representativa da população masculina da cidade de São Paulo, a pesquisa se propôs a comparar os diferentes estratos sociais. A amostra foi selecionada através do critério de cotas das faixas etárias, cuja distribuição obedeceu àquela encontrada no último censo (SEADE, 1994). A distribuição das entrevistas em seis áreas geográficas do municí- 
pio (SEADE, 1994) foi controlada, evitando-se a concentração de entrevistados com residência em poucas áreas. Ou seja, a cada entrevistadora era dada uma cota de entrevistas a ser realizadas de acordo com a idade e a área geográfica de residência do entrevistado. Considerou-se também a ocupação atual como critério de inclusão para entrevista e para tal, foi usada a classificação de inserção profissional da Organização Internacional do Trabalho (OIT). A amostra inicial de 600 homens foi diminuída para 597, pois três entrevistas foram excluídas. As características da amostra planejada e realizada estão expostas na Tabela 1 .

Os participantes, abordados em locais públicos ou em seu local de trabalho, foram convidados a participar do estudo, caso os critérios de seleção fossem preenchidos. As entrevistas foram realizadas nos locais de abordagem, como centros comerciais, cinemas, feiras ou local de trabalho. Do total de homens abordados $11 \%$ recusaram ser entrevistados. A duração de cada entrevista foi de aproximadamente 45 minutos. Nas perguntas mais íntimas tomou-se o cuidado de utilizar cartões para a resposta. Ou seja, as opções para resposta foram apresentadas por escrito ao entrevistado que respondia pelo número da opção escolhida. Nessas perguntas utilizou-se linguagem coloquial que não permitisse dúvidas.

Por exemplo, para perguntar sobre orientação sexual utilizamos a seguinte questão: "Em termos de atração sexual, o senhor diria: (mostrar o cartão) 1) Ao longo de minha vida sempre me senti atraído por mulheres; 2) algumas vezes me senti atraído por homens; 3) sempre me senti atraído por homens e mulheres igualmente; 4) só me senti atraído por homens". Sobre a experiência sexual anterior foi perguntado: "Em termos de relações sexuais (mostrar o cartão): 1) Só tive relações com mulheres; 2) já tive relações com homens; 3) já tive relações com homens e mulheres". Como estávamos interessados em explorar as múltiplas parcerias sexuais perguntamos ao entrevistado: "Como o senhor definiria a sua situação sexual atualmente? (mostrar o cartão): 1) Tenho uma parceira e só tenho relações com ela; 2 ) tenho uma parceira e às vezes tenho relação com outras mulheres; 3) tenho uma parceira e às vezes tenho relação com outras pessoas, homens ou mulheres; 4) tenho mais de uma parceira e só tenho relações com elas; 5) não tenho parceira e só tenho relações com mulheres; 6) não tenho parceira e tenho relações com homens e mulheres".

Todos os questionários foram revisados pela equipe de campo e a confirmação da realização da entrevista foi feita pelo telefone com $20 \%$ dos entrevistados sorteados.
Tabela 1

Critérios utilizados para seleção da amostra de homens de 21 a 50 anos. Município de São Paulo, 1997.

\begin{tabular}{|c|c|c|c|c|}
\hline Critério & $\%$ & $\begin{array}{l}\text { Entrevistas } \\
\text { planejadas }\end{array}$ & $\begin{array}{l}\text { Entrevistas } \\
\text { realizadas }\end{array}$ & $\%$ \\
\hline \multicolumn{5}{|l|}{ Faixa etária 1} \\
\hline $21-29$ & 38,37 & 230 & 246 & 41,2 \\
\hline $30-39$ & 36,03 & 216 & 211 & 35,3 \\
\hline $40-50$ & 25,60 & 154 & 140 & 23,4 \\
\hline \multicolumn{5}{|c|}{ Níveis de inserção no trabalho 2} \\
\hline Alto & 12,2 & 73 & 87 & 14,5 \\
\hline Médio & 42,6 & 256 & 252 & 42,2 \\
\hline Baixo & 45,2 & 271 & 258 & 43,2 \\
\hline \multicolumn{5}{|l|}{ Zonas geográficas 3} \\
\hline Centro & 13,03 & 78 & 99 & 16,5 \\
\hline Norte & 20,31 & 122 & 120 & 20,1 \\
\hline Oeste & 4,44 & 27 & 33 & 5,5 \\
\hline Sul & 22,43 & 134 & 110 & 18,4 \\
\hline Sudeste + Leste & 39,70 & 239 & 235 & 39,3 \\
\hline
\end{tabular}

1 IBGE, Censo demográfico de 1991.

2 Ação Educativa, Assessoria, Pesquisa e Informação, 1996.

3 Fundação SEADE, Município de São Paulo. Projeção da população residente. São Paulo, 1994

Em relação ao estrato social os entrevistados foram classificados pelos critérios da ABA/ ABIPEME (Associação Brasileira de Anunciantes/Associação Brasileira de Pesquisa de Mercado) (Eduardo et al., 1979). Este critério, criado em 1979, pontua a educação do chefe da família e os bens de consumo no domicílio do entrevistado para formar a categoria de classes que são estratificadas em A, B, C, D e E.

Os dados quantitativos foram computados e analisados através do programa estatístico para Ciências Sociais (SPSS/PC+) (SPSS Incorporation, 1994) As técnicas estatísticas utilizadas para testar hipóteses foram o teste do quiquadrado com nível de significância de 0,05 para rejeitar a hipótese de não associação e o teste de $\mathrm{t}$ de comparação das médias.

\section{Resultados}

\section{Caracterização sócio-demográfica}

A maioria dos 597 homens entrevistados tinha até 29 anos. A média de idade encontrada foi de 32,7 e a mediana 31 anos (Tabela 1).

A maioria (72\%) dos entrevistados tinha pelo menos o primeiro grau completo e quase metade (48\%) colegial ou curso superior com- 
pleto ou incompleto. Em relação ao estrato social a maioria foi classificada como classes B ou C, um quinto deles como classe D e uma pequena proporção inferior ou por volta de $5 \%$ como A ou E. Para fins de análise foram agrupadas as classe A e B e as classes D e E. A renda familiar per capita foi calculada e quase metade dos entrevistados apresentou uma renda maior do que cinco ou mais salários mínimos. Os entrevistados apresentaram uma grande diversidade de ocupações, sendo que a maioria (52\%) trabalhava no setor de comércio ou serviços, como funcionários ou pequenos proprietários (Tabela 2).

Podemos caracterizar, portanto, a amostra estudada como uma população adulta, escolarizada, inserida no mercado de trabalho e com renda mensal acima da média da população (SEADE, 1994).

\section{Orientação sexual}

Quase a totalidade dos entrevistados, 96,5\% (576), afirmou sempre ter se sentido atraído por mulheres, $2 \%$ (13) declararam atração por homens e mulheres e 1\% (7) só se sentem atraídos por homens (um não respondeu).

As respostas sobre a experiência sexual anterior apresentam porcentuais ligeiramente distintos das respostas sobre orientação sexual: $94,5 \%$ (564) afirmaram só terem tido relações sexuais com mulheres, $2 \%$ (10) apenas com homens e 3,5\% (21) com homens e mulheres.

\section{União sexual}

Perguntado sobre o seu estado conjugal, mais da metade dos homens, 54\% (325) coabitavam com uma companheira em união legal ou consensual, 18\% (107) eram solteiros e estavam sem parceira sexual fixa no momento da entrevista, 16\% (97) eram solteiros, mas tinham uma parceira sexual fixa com a qual não coabitavam, $6 \%$ (36) eram separados ou divorciados e estavam sem parceira no momento da entrevista, 5\% (32) eram separados e tinham parceira sexual, com a qual não coabitavam. Para efeito de análise consideramos os homens separados sem parceira como solteiros sem parceira fixa, assim como os separados com parceira, mas sem coabitação, foram considerados solteiros na mesma condição. Dos 325 homens que coabitavam com uma mulher, $79 \%$ (256) estavam no primeiro casamento.

\section{Tabela 2}

Características sócio-demográficas dos homens de 21 a 50 anos entrevistados. Município de São Paulo, 1997.

\begin{tabular}{|c|c|c|}
\hline $\begin{array}{l}\text { Características } \\
\text { sócio-demográficas }\end{array}$ & $\mathrm{n}$ & $\%$ \\
\hline \multicolumn{3}{|l|}{ Escolaridade } \\
\hline Nenhuma & 8 & 1,3 \\
\hline Primário incompleto & 34 & 5,6 \\
\hline Primário completo & 48 & 8,0 \\
\hline 1o grau incompleto & 79 & 13,2 \\
\hline 1o grau completo & 75 & 12,5 \\
\hline 2o grau incompleto & 63 & 10,5 \\
\hline 2o grau completo & 114 & 19,0 \\
\hline Superior incompleto & 78 & 13,0 \\
\hline Superior completo & 97 & 16,2 \\
\hline Total1 & 596 & 100,0 \\
\hline \multicolumn{3}{|l|}{ Estrato social } \\
\hline Classe A & 22 & 3,7 \\
\hline Classe B & 198 & 33,4 \\
\hline Classe C & 224 & 37,8 \\
\hline Classe D & 115 & 19,4 \\
\hline Classe $\mathrm{E}$ & 34 & 5,7 \\
\hline Total2 & 593 & 100,0 \\
\hline \multicolumn{3}{|l|}{ Renda familiar per capita } \\
\hline Menos de 1 salário mínimo* & 20 & 3,4 \\
\hline 1 a 2 salários mínimos & 92 & 15,4 \\
\hline 2 a 3 salários mínimos & 98 & 16,4 \\
\hline 3 a 5 salários mínimos & 124 & 20,8 \\
\hline 5 ou mais salários mínimos & 262 & 44,0 \\
\hline Total 3 & 596 & 100,0 \\
\hline \multicolumn{3}{|l|}{ Ocupação** } \\
\hline $\begin{array}{l}\text { Funcionários de comércio } \\
\text { e serviços }\end{array}$ & 163 & 27,0 \\
\hline Pequenos proprietários & 77 & 13,0 \\
\hline Trabalhadores de escritório & 73 & 12,0 \\
\hline Operários & 64 & 11,0 \\
\hline Gerentes & 63 & 10,0 \\
\hline Técnicos não especializados & 59 & 10,0 \\
\hline Profissionais liberais & 55 & 9,0 \\
\hline Técnicos especializados & 23 & 4,0 \\
\hline Outras & 20 & 3,0 \\
\hline Total 4 & 597 & 100,0 \\
\hline
\end{tabular}

1 Não respondeu;

24 não responderam;

31 não respondeu;

* Valor em reais na época $\mathrm{R} \$ 112,00$.

** Prandi, s.d. 


\section{Vida sexual atual}

Mais da metade dos entrevistados, 59\% (344), referiram ter apenas uma parceira e só manter relações sexuais com ela, 22,5\% (131) não tinham parceira no momento da entrevista, e 18\% (107) tinham múltiplas parceiras (destes, 94 homens relataram ter uma parceira e ocasionalmente se relacionar sexualmente com outras mulheres e 13 relataram ter mais de uma parceira e só se relacionar com elas). Uma minoria se revelou bissexual: $1 \%$ (7) referiram se relacionar com homens e mulheres, destes, 0,8\% (5) não tinham parceiros/as no momento. Quinze homens não responderam a esta questão.

As respostas foram agrupadas em três categorias: sem parceiro/a, com um parceiro e múltiplos parceiros. A associação com outras variáveis foi testada, como mostra a Tabela 3.

Encontrou-se associação entre a idade atual do entrevistado e parceria sexual. Existe maior proporção de homens com múltiplas parceiras sexuais na faixa etárias de 40 a 50 anos, 25\%, enquanto a porcentagem de múltiplas parceiras nos outros grupos etários 21 a 29 anos e 30 a 39 encontrada foi respectivamente $16 \%$ e $18 \%$. A diferença é estatisticamente significante $(\mathrm{p}=0,00000)$

Também observou-se associação entre escolaridade e parceria sexual. Dos homens que cursaram até a quarta série $29 \%$ tinham múltiplas parceiras, enquanto dos que haviam cursado curso superior apenas $16 \%$ tinham múltiplas parceiras, sendo esta diferença estatisticamente significante $(\mathrm{p}=0,02)$.

Não foi encontrada associação entre parceria sexual e estratificação social $(p>0,05)$. Ao analisar apenas os homens com parceira sexual, também não foi encontrada associação estatística entre parceria e coabitação ( $p>0,05)$.

Excluindo-se os homens sem parceria sexual encontrou-se associação entre não usar

Estrato social, escolaridade, idade, coabitação e uso do preservativo entre homens de 21 a 50 anos entrevistados, de acordo com parceria sexual. Município de São Paulo, 1997

\begin{tabular}{|c|c|c|c|c|c|c|c|c|}
\hline & \multicolumn{2}{|c|}{ Sem parceira } & \multicolumn{2}{|c|}{ Parceira única } & \multicolumn{2}{|c|}{ Múltiplas parceiras } & \multirow[t]{2}{*}{ n total } & \multirow[t]{2}{*}{$\mathrm{p}$} \\
\hline & $n$ & $\%$ & $\mathrm{n}$ & $\%$ & $n$ & $\%$ & & \\
\hline \multicolumn{9}{|l|}{ Idade 1} \\
\hline Até 29 anos & 79 & 33,4 & 121 & 51 & 37 & 15,6 & 237 & 0,00000 \\
\hline 30 a 39 anos & 33 & 15,9 & 137 & 66,2 & 37 & 17,9 & 207 & \\
\hline 40 a 50 anos & 17 & 12,4 & 86 & 62,8 & 34 & 24,8 & 137 & \\
\hline \multicolumn{9}{|l|}{ Escolaridade 2} \\
\hline 4으 série & 18 & 20,0 & 46 & 51,1 & 26 & 28,9 & 90 & 0,02394 \\
\hline 8o série & 25 & 16,9 & 91 & 61,5 & 32 & 21,6 & 148 & \\
\hline 2o grau & 40 & 23,4 & 108 & 63,2 & 23 & 13,5 & 171 & \\
\hline Superior & 46 & 26,9 & 98 & 57,3 & 27 & 15,8 & 171 & \\
\hline \multicolumn{9}{|l|}{ Estrato social 3} \\
\hline $\mathrm{A} / \mathrm{B}$ & 42 & 19,5 & 136 & 63,3 & 37 & 17,2 & 215 & 0,062051 \\
\hline C & 50 & 23 & 126 & 58,1 & 41 & 18,9 & 217 & \\
\hline $\mathrm{D} / \mathrm{E}$ & 36 & 24,8 & 80 & 55,2 & 29 & 20 & 145 & \\
\hline \multicolumn{9}{|l|}{ Coabitação4 } \\
\hline Não & * & & 90 & 70 & 38 & 30 & 128 & 0,06096 \\
\hline Sim & * & & 254 & 79 & 69 & 21 & 323 & \\
\hline \multicolumn{9}{|c|}{ Uso da preservativo 5} \\
\hline Não usam & * & & 230 & 67 & 47 & 44 & 277 & 0,00000 \\
\hline Usam extra & * & & 55 & 16 & 40 & 37 & 95 & \\
\hline Com parceira & * & & 59 & 17 & 20 & 19 & 79 & \\
\hline
\end{tabular}

1 Total $=581$ (16 não responderam); 2 Total $=580$ (17 não responderam);

3 Total $=577$ (20 não responderam); 4 Total $=451 ; 5$ Total $=451$.

* Sem parceira foram excluídos do teste. 
preservativo e ter uma única parceira, $67 \%$ dos homens com apenas uma parceira sexual referiram não usar o preservativo, enquanto $44 \%$ dos homens com múltiplas parceiras referem não usar o preservativo ( $\mathrm{p}=0,00000)$.

Considerando-se apenas os homens com parceira sexual e analisando-os por faixa etária, foi observada associação entre não coabitar e ter múltiplas parceiras sexuais apenas para os da faixa etária inferior a 29 anos $(\mathrm{p}=0,04483)$ (Tabela 4).

\section{Número de parceiras no ano anterior}

Perguntados sobre o número de parceiras no ano anterior, a maioria dos homens, 59\%, (342) referiu ter tido apenas uma parceira sexual, $24 \%$ (144) tiveram entre duas ou três parceiras, $16 \%$ (92) tiveram quatro ou mais parceiras e $3 \%$ (19) não responderam a esta questão. Como era esperado, os homens solteiros sem parceira fixa são os que tiveram maior número de parceiras no ano anterior (média 4,45), comparado aos casados (média 1,48) e solteiros com uma parceira fixa (média 3,03). As diferenças entre estes grupos, testadas utilizando-se o teste de $\mathrm{t}$ de comparação das médias, mostraram-se estatisticamente significante $(p=0,000 ; p=0,05$ e p $=0,01$ respectivamente). Enquanto $78 \%$ dos homens casados tiveram uma só parceira no ano anterior, para os solteiros com parceira fixa, mas sem coabitação, este percentual cai para $48 \%$, e $21 \%$ para os solteiros sem parceira.

\section{Conhecimento sobre AIDS}

Seis perguntas foram utilizadas para avaliar o conhecimento dos entrevistados sobre AIDS. A primeira explorava o conhecimento sobre os modos de transmissão do HIV através de respostas espontâneas e múltiplas, enquanto as cinco perguntas restantes abordavam algumas características da infeção ou doença. A maioria dos entrevistados possui alto conhecimento sobre a transmissão da AIDS.

Perguntados como a AIDS se transmite apenas $6 \%$ responderam que beijando na boca, $97 \%$ pelas relações sexuais, $47 \%$ através de transfusão de sangue, $73 \%$ por uso de seringas e agulhas, $0,3 \%$ através de utensílios domésticos, $6,2 \%$ na doação de sangue. A proporção de respostas erradas é bastante baixa. Não foram citadas respostas como: que a AIDS pode ser transmitida por contato físico não sexual, pela picada do mosquito, ou pelo assento do vaso sanitário, etc. Quase 50\% dos entrevistados mencionaram a transfusão de sangue como possibilidade de contrair o HIV.

As cinco perguntas restantes que abordaram algumas características da infecção ou da doença são mostradas na Tabela 5. Estas perguntas foram computadas para construir uma escala de conhecimento, na qual $74 \%$ (442) dos entrevistados mostraram ter alto conhecimento sobre AIDS, $22 \%$ (130) médio conhecimento e apenas $4 \%$ (24) têm baixo conhecimento da doença.

Tabela 4

Homens entrevistados de 21 a 50 anos, com parceiras sexuais classificados por coabitação

e tipo de parceria controlados por faixa etária. Município de São Paulo,1997.

\begin{tabular}{|c|c|c|c|c|c|}
\hline Faixa etária & Tipo de parceria & $\begin{array}{l}\text { Parceira } \\
\text { única }\end{array}$ & $\begin{array}{l}\text { Múltiplas } \\
\text { parceiras }\end{array}$ & Total & $\mathbf{p}$ \\
\hline \multirow[t]{2}{*}{21 a 29 anos } & Solteiro com parceira sem coabitação & $59(70,2 \%)$ & $25(29,8 \%)$ & 84 & \\
\hline & Morando com parceira & $62(83,8 \%)$ & $12(16,2 \%)$ & 74 & \\
\hline Total & & 121 & 37 & 158 & 0,04483 \\
\hline \multirow[t]{2}{*}{30 a 39 anos } & Solteiro com parceira sem coabitação & $26(72,2 \%)$ & $10(27,8)$ & 36 & \\
\hline & Morando com parceira & $111(81 \%)$ & $26(19 \%)$ & 137 & \\
\hline Total & & 137 & 36 & 173 & 0,24710 \\
\hline \multirow[t]{2}{*}{40 a 50 anos } & Solteiro com parceira sem coabitação & $5(62,5 \%)$ & $3(37,5 \%)$ & 8 & \\
\hline & Morando com parceira & $81(72,3 \%)$ & $31(27,7 \%)$ & 112 & \\
\hline Total & & 86 & 34 & 120 & 0,55146 \\
\hline
\end{tabular}


Foram encontradas associações entre o grau de conhecimento sobre AIDS, escolaridade e estrato social. Entre os homens que estudaram até a quarta série $57 \%$ tinham alto conhecimento sobre AIDS, enquanto $79 \%$ dos que tinham nível superior demostraram alto conhecimento sobre AIDS ( $p=0,00017$ ). Entre os entrevistados classificados em classes A e B, $84 \%$ tinham alto conhecimento sobre AIDS, enquanto $55 \%$ dos classificados em classe D e E mostraram alto conhecimento sobre AIDS ( $p=0,00000)$. Perguntado se o entrevistado conhecia pessoalmente alguém com AIDS, 63\% responderam que sim.

\section{Conhecimento das DST}

Duas perguntas avaliaram o conhecimento sobre DST dos entrevistados. Uma que pedia ao entrevistado para citar as doenças que conhecia. Em seguida, perguntava-se se o entrevistado já tinha ouvido falar sobre as DST não mencionadas. As DST mais lembradas espontaneamente foram a gonorréia (83\%), a AIDS (71\%) e a sífilis (58\%). Quando a pergunta foi estimula$\mathrm{da}$, as mais conhecidas foram a AIDS (99\%), a gonorréia (98\%), a sífilis (89\%) e o cancro mole $(74,5 \%)$. Para as respostas espontâneas o conhecimento de AIDS, gonorréia, sífilis, condiloma e herpes estão associados com aumento de escolaridade $(\mathrm{p}<0.01)$. Para as respostas estimuladas o conhecimento sobre AIDS não se encontra associado à escolaridade, quase $100 \%$ dos entrevistados já ouviram falar da AIDS.

Tal como havia sido feito com o conhecimento sobre a AIDS, estas respostas foram computadas em uma escala, mostrando baixo conhecimento em relação às DST para 33,5\% (198) dos entrevistados, médio conhecimento para $46 \%$ (270) e alto conhecimento para $21 \%$ (122) dos entrevistados.

Foram encontradas associações entre conhecimento de DST com escolaridade e classe social. Dos entrevistados que estudaram até a quarta série apenas 5\% mostraram alto conhecimento sobre DST, enquanto dos que fizeram curso superior $35 \%$ tinham alto conhecimento ( $p=0,00000)$. Entre os homens classificados entre A e B $30 \%$ mostraram alto conhecimento das DST, enquanto menos de $10 \%$ dos homens classificados entre D e E tinham alto conhecimento $(\mathrm{p}=0,00000)$.

Tabela 5

Conhecimento das características da infecção/doença.

\begin{tabular}{|c|c|c|c|c|}
\hline \multirow{2}{*}{$\begin{array}{l}\text { Características da doença } \\
\text { 1) É possível a pessoa ter aspecto }\end{array}$} & \multicolumn{2}{|c|}{ População estudada } & \multicolumn{2}{|c|}{ PNDS* } \\
\hline & Sim & $561(94 \%)$ & Sim & $91,2 \%$ \\
\hline \multirow[t]{2}{*}{ saudável e ser portadora da AIDS } & Não & $30(5 \%)$ & Não & $3,9 \%$ \\
\hline & Não sabe & $6(1 \%)$ & Não sabe & $4,9 \%$ \\
\hline \multirow[t]{3}{*}{ 2) A AIDS tem cura** } & $\operatorname{Sim}$ & $33(5,5 \%)$ & $\operatorname{Sim}$ & $2,8 \%$ \\
\hline & Não & $550(92,1 \%)$ & Não & $95,1 \%$ \\
\hline & Não sabe & $13(2,2 \%)$ & Não sabe & $1,8 \%$ \\
\hline 3) A AIDS pode ser transmitida & Sim & $571(95,6 \%)$ & Sim & $91,9 \%$ \\
\hline \multirow[t]{2}{*}{ de mãe para o bebêe $\hat{e}^{\star \star \star}$} & Não & $9(1,5 \%)$ & Não & $2,5 \%$ \\
\hline & Não sabe & $13(2,2 \%)$ & Não sabe & $5,6 \%$ \\
\hline 4) Há algo que a pessoa possa fazer & $\operatorname{Sim}$ & $587(98,3 \%)$ & $\operatorname{Sim}$ & 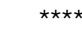 \\
\hline \multirow[t]{2}{*}{ para se proteger da AIDS } & Não & $3(0,5 \%)$ & Não & 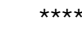 \\
\hline & Não sabe & $7(1,2 \%)$ & Não sabe & 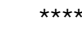 \\
\hline \multirow[t]{4}{*}{ 5) A AIDS é uma doença fatal } & Quase sempre & $526(88,1 \%)$ & Quase sempre & $89,8 \%$ \\
\hline & Quase nunca & $7(1,2 \%)$ & Quase nunca & $1,8 \%$ \\
\hline & Às vezes & $62(10,4 \%)$ & Às vezes & $7,4 \%$ \\
\hline & Não sabe & $2(0,3 \%)$ & Não sabe & $0,7 \%$ \\
\hline
\end{tabular}

* Dados do Estado de São Paulo, PNDS-1996, Badiani et al. (1997).

$\star \star$ Não respondeu $=1$.

$\star \star \star$ Não responderam $=4$

$\star \star \star \star$ Não disponível. 
Não foi encontrada associação entre idade e conhecimento de DST, seja por respostas estimuladas ou espontâneas.

\section{Percepção de risco de contrair o HIV}

A percepção de risco foi avaliada através de duas perguntas. Uma que solicitava ao entrevistado para auto classificar o risco de contrair o HIV. A maioria dos homens, 46\% (273), respondeu riscos pequenos, $26 \%$ (156) referem nenhum risco, quase $18 \%$ (107) responderam riscos moderados e 9,5\% (57) referem riscos grandes. Menos de 1\% (três homens) responderam não saber sobre seu risco e um homem referiu ter AIDS.

A segunda pergunta questionava o motivo da auto-classificação do risco. Dentre os homens que referiram nenhum risco ou risco pequeno ( $72 \%, \mathrm{n}=429$ ), 53\% (226) tinham apenas uma parceira ou haviam limitado o número de parceiras; 25\% (106) usavam o preservativo, $9 \%$ (38) disseram que poderia acontecer um acidente e serem contaminados, $5 \%$ (20) confiavam na parceira, pois a conheciam bem, $3 \%$ (13) poderiam ter ou ter tido relação com alguém que não sabiam ser $\mathrm{HIV+,} \mathrm{3 \%} \mathrm{(11)} \mathrm{não}$ usavam drogas injetáveis, não receberam transfusão de sangue e não tiveram contato com homossexuais. Apenas 2\% (10) referiram outras respostas tais como: "não convive com quem tem AIDS", "tem fé, toma cuidado", "fez exame recentemente" ou "não acredita que AIDS exis$t a$ ". Menos de $1 \%$ (5) estavam sem parceira no momento da entrevista.

Entre os 27\% (164) dos homens que se auto classificaram com risco moderado ou grande, perguntados sobre os motivos afirmaram: $26 \%$ (43) poderiam vir a precisar de uma transfusão de sangue, $14 \%$ (23) poderiam vir a ter uma relação com alguém contaminado sem saber, $11 \%$ (18) tinham ou tiveram muitas parceiras, $10 \%$ (16) não usavam camisinha, 8\% (13) devido à profissão, $7 \%$ (12) sem parceira fixa, $7 \%$ (12) a camisinha não é totalmente confiável, $7 \%$ (11) referiram que poderiam se contaminar em um acidente, 1\% (2) uso de droga injetável, $2 \%$ (3) referem motivos religiosos e místicos como ser punido por Deus, $2 \%$ (4) referiram que a parceira tinha outro parceiro e $4 \%$ (7) deram outras respostas tais como: "qualquer um pode ter AIDS hoje", "não existe fiscalização em saúde pública", "posso ter pego fazendo sexo oral", etc.

Maior percepção de risco foi encontrada associada com estrato social mais alto, maior escolaridade, maior conhecimento de DST, múltiplas parceiras, não coabitar com a parceira, maior uso de preservativo e maior número de parceiras no ano anterior. Não foi encontrada associação entre maior conhecimento sobre AIDS e maior percepção de risco, assim como também não foi encontrada com idade (Tabela 6$)$.

\section{Prevenção da AIDS}

Perguntados como evitar a AIDS, com possibilidade de múltiplas respostas, 86,5\% (517) responderam que através do uso de preservativos, $36 \%$ (214) do uso de seringas e agulhas descartáveis, 25\% (149) do cuidado com transfusões de sangue, $17 \%$ (101) ter uma só parceira sexual, 15\% (89) diminuir o número de parceiras, 13\% (77) não usar drogas, 5\% (29) praticar sexo seguro, 4\% (23) abster-se de relações sexuais e $3 \%$ (18) responderam que não tendo relações homossexuais. Sobre o que significava sexo seguro, 65\% (388) responderam que era sexo com preservativo e $55 \%$ (328) que era sexo com uma parceira conhecida (respostas múltiplas).

\section{Uso do preservativo masculino}

Considerando todos os entrevistados, 17\% (98) deles responderam que utilizavam o preservativo em relações extra conjugais, 18\% (109) homens solteiros sem parceiras disseram que estavam habituados a usar o preservativo, enquanto $13 \%$ (80) disseram que utilizavam com a parceira, 38\% (228) homens referiram não usar preservativo e 13\% (82) utilizam ocasionalmente com a parceira fixa ou outra mulher. Para efeito de análise consideramos o uso ocasional de preservativo como não uso.

O preservativo é mais utilizado entre os homens sem parceira fixa ou solteiros. Entre os 325 homens que coabitavam, 16\% (52) referiram usar preservativo em relações extra maritais, $11 \%$ (36) usavam com a parceira e $73 \%$ (237) disseram não usar. Dos 129 homens que têm parceiras as não coabitavam com ela, 33\% (43) referem usar preservativo em uma relação com uma parceira diferente da parceira fixa, $33 \%$ (43) usavam com a companheira e 33\% (43) disseram não usar. Dos 143 homens que estavam sem parceira no período da entrevista $76 \%$ (109) usaram quando tiveram uma relação sexual, os restantes $20 \%$ (29) disseram que não usavam, e 3\% (5)dos homens não responderam.

O uso do preservativo masculino foi encontrado associado à idade, sendo mais utilizado pelos homens jovens $(p=0,0000)$. De fato, para a faixa etária mais elevada, 40 a 50 anos, $70 \%$ dos homens disseram não usar. Entre os de 30 a 39 anos, este percentual decresce para $60 \%$, para os que têm entre 20 e 29 anos, para $34 \%$. 
O uso do preservativo aumenta com a escolaridade. Afirmaram não usar o preservativo $61 \%$ dos homens que estudaram até a quarta série, $58 \%$ dos que estudaram até a oitava série, $50 \%$ dos que têm até o colegial e $39 \%$ dos que têm nível superior de escolaridade $(\mathrm{p}=$ 0,01288). Embora entre os homens com múltiplas parceiras houvesse uma maior proporção que disse usar o preservativo do que entre os homens com uma parceira, uma grande porcentagem (44\%) disse ter múltiplas parceiras e não usar o preservativo (Tabela 3 ).

Os homens que disseram usar o preservativo têm maior percepção de risco do que aqueles que disseram não usar, sendo esta diferença estatisticamente significante $(p=0,00013)$ (Tabela 6).

\section{Discussão}

\section{Orientação sexual}

Os dados relativos à orientação e experiência sexual contrariam as crenças, amplamente disseminadas, a respeito da bissexualidade, da multiplicidade de parceria e da infidelidade do homem brasileiro, o que é particularmente relevante no caso da AIDS. De fato, nos primórdios da disseminação da epidemia entre mulheres foi sugerido que a ponte entre a AIDS e a população feminina fossem os homens bissexuais (Guimarães, 1994). Embora naquele momento essa categoria estivesse presente num percentual importante dos casos de AIDS entre homens, parece que a feminilização da epidemia tem estado mais relacionada a comportamentos heterossexuais. Apesar desta não ser uma amostra representativa dos homens do Município de São Paulo, vale ressaltar que os percentuais de homo e bissexualidade encontrados nesta amostra de homens da cidade de São Paulo são similares aos encontrados em vários países, tais como mostram recentes pesquisas de comportamento sexual: Estados Unidos 3,9\% (Laumann et al., 1994); Europa (Hubert et al., 1998): Bélgica 6,1\% (1993); Finlândia 4,9\% (1992); Alemanha 4,4\% (1990); Noruega 5,2\% (1992); Portugal 0,9\% (1991), Espanha 7,3\% (1990) Suíça 4,7\% (1992) e França 4,1\% (1992) (Leridon et al., 1994).

\section{Múltiplas parcerias}

Os dados sugerem uma tendência de monogamia para homens em coabitação, principalmente os mais jovens. Talvez haja uma tendência para a monogamia seriada, mas os dados
Tabela 6

Percepção de risco por classe social, escolaridade, idade, conhecimento de DST/AIDS, parceria sexual, coabitação, uso de preservativo e número de parceiras entre homens. Município de São Paulo, 1997

\begin{tabular}{|c|c|c|c|c|c|c|c|}
\hline & \multicolumn{2}{|c|}{ Sem risco } & \multicolumn{2}{|c|}{$\begin{array}{l}\text { Risco } \\
\text { pequeno }\end{array}$} & \multicolumn{2}{|c|}{$\begin{array}{l}\text { Risco moderado/ } \\
\text { grande }\end{array}$} & \multirow[t]{2}{*}{$p$} \\
\hline & $\mathrm{n}$ & $\%$ & $\mathrm{n}$ & $\%$ & $n$ & $\%$ & \\
\hline \multicolumn{8}{|l|}{ Classe social 1} \\
\hline $\mathrm{A} / \mathrm{B}$ & 47 & 30 & 116 & 43 & 55 & 34 & 0,03085 \\
\hline C & 59 & 38 & 95 & 35 & 69 & 42 & \\
\hline$D / E$ & 50 & 32 & 59 & 22 & 39 & 24 & \\
\hline \multicolumn{8}{|l|}{ Escolaridade 2} \\
\hline 4a série & 34 & 22 & 34 & 12,5 & 20 & 12 & 0,00130 \\
\hline 8a série & 47 & 30 & 63 & 23 & 44 & 27 & \\
\hline 2o grau & 48 & 31 & 78 & 28,5 & 51 & 31 & \\
\hline Superior & 26 & 17 & 98 & 36 & 49 & 30 & \\
\hline \multicolumn{8}{|l|}{ Idade $^{3}$} \\
\hline $21-29$ & 59 & 38 & 106 & 38,8 & 79 & 48,2 & 0,31581 \\
\hline $30-39$ & 59 & 38 & 100 & 36,6 & 50 & 30,5 & \\
\hline $40-50$ & 38 & 24 & 67 & 24,5 & 35 & 21,3 & \\
\hline \multicolumn{8}{|c|}{ Conhecimento AIDS 4} \\
\hline Baixo & 10 & 6 & 9 & 3,3 & 4 & 2,4 & 0,16404 \\
\hline Médio & 34 & 22 & 53 & 19,4 & 43 & 26,8 & \\
\hline Alto & 112 & 72 & 211 & 77,3 & 117 & 71,3 & \\
\hline \multicolumn{8}{|c|}{ Conhecimento DST 5} \\
\hline Baixo & 67 & 43 & 75 & 27 & 54 & 33 & 0,02720 \\
\hline Médio & 65 & 42 & 129 & 48 & 75 & 46 & \\
\hline Alto & 24 & 15 & 64 & 23 & 34 & 21 & \\
\hline \multicolumn{8}{|l|}{ Parceria Sexual6 } \\
\hline Sem parceira & 26 & 20 & 48 & 37 & 55 & 43 & 0,00003 \\
\hline 1 parceira & 106 & 30 & 165 & 48 & 72 & 21 & \\
\hline Múltiplas & 20 & 19 & 54 & 51 & 32 & 30 & \\
\hline \multicolumn{8}{|l|}{ Coabitação7 } \\
\hline Sem parceira & 29 & 20 & 53 & 38 & 59 & 42 & 0,00004 \\
\hline Não coabita & 25 & 19,4 & 69 & 53,5 & 35 & 27,1 & \\
\hline Coabita & 102 & 31,6 & 151 & 46,7 & 70 & 21,7 & \\
\hline \multicolumn{8}{|c|}{ Uso de preservativo 8} \\
\hline Não usa & 98 & 62,8 & 140 & 51,5 & 69 & 42 & 0,00013 \\
\hline Extra marital & 13 & 8,3 & 57 & 21 & 26 & 16 & \\
\hline Usa parceira & 24 & 15,4 & 31 & 11,4 & 25 & 15 & \\
\hline S/parceira & 21 & 13,5 & 44 & 16,2 & 44 & 27 & \\
\hline \multicolumn{8}{|c|}{$\begin{array}{l}\text { Número de parceiras } \\
\text { no ano anterior }{ }^{9}\end{array}$} \\
\hline Uma & 111 & 71 & 174 & 64 & 86 & 52 & 0,00104 \\
\hline Duas/três & 31 & 20 & 69 & 25 & 42 & 26 & \\
\hline Quatro ou + & 14 & 9 & 30 & 11 & 36 & 22 & \\
\hline
\end{tabular}

1 Não responderam $=8 ; 2$ Não responderam $=5 ; 3$ Não responderam $=4$;

4 Não responderam $=4 ; 5$ Não responderam $=10 ; 6$ Não responderam $=19 ;$

7 Não responderam $=4 ; 8$ Não responderam $=5 ; 9$ Não responderam $=4$. 
não permitem esta conclusão. A comparação entre os grupos etários aponta para uma provável mudança de comportamento nos homens que iniciaram a vida sexual no advento da AIDS em relação aos homens de gerações anteriores. Estudos de acompanhamento da epidemia em alguns locais específicos mostram uma tendência ascendente na faixa etária de 35 a 39 anos a partir de 1993 (Menesia, 1999), sugerindo uma menor auto proteção dos homens destas faixas etárias. Dois pontos devem ser ressaltados na maior vulnerabilidade dos homens mais velhos: maior porcentagem de homens com múltiplas parcerias e menor uso de preservativo.

\section{Conhecimento DST/AIDS}

Tal como já havia sido apontado em estudos anteriores (Loyola,1994; Badiani et al., 1997) o conhecimento sobre AIDS é bastante disseminado na população, assim como suas formas de transmissão e algumas características da doença. Apesar deste ser um conhecimento parcial e fragmentário, podemos afirmar que tem sido bem sucedida a disseminação dos perigos e formas de prevenção da doença pela mídia e campanhas governamentais e não-governamentais.

Comparando-se os resultados deste estudo com aqueles obtidos pela PNDS-1996, observamos que os diferenciais são menores do que 5\% em quatro perguntas da Tabela 3 . Em relação a conhecer alguém com AIDS, a diferença entre o resultado da PNDS-1996 e este estudo é bem maior, tendo sido encontrado $48,8 \%$ na PNDS e $63,3 \%$ neste estudo (Badiani et al., 1997). Isto se deve provavelmente ao fato do campo desta pesquisa ter sido o Município de São Paulo, enquanto a base amostral para São Paulo utilizado na PNDS é o estado. Além disso, a amostra deste estudo não é causal. Tal como encontrado na PNDS-1996, maior conhecimento sobre as características da AIDS está associado à maior escolaridade. Podemos então verificar concordância de nossos resultados com os dados da PNDS, em que pesem as diferenças amostrais.

Sobre o conhecimento da AIDS, suas características e formas de transmissão o estudo confirma o alto conhecimento sobre a transmissão da doença, exceto na questão da transfusão de sangue citada por quase metade dos entrevistados (47\%). Em pesquisa realizada no Rio de Janeiro, Loyola (1994) encontrou 19,4\%. No componente qualitativo do estudo a autora ressalta que "o temor de necessitar de uma transfusão de sangue continua sendo o principal receio dos informantes como possibilidade de contaminação pelo vírus HIV" (Loyola, 1994:69). Considerando-se que desde 1986 existe controle do san- gue e hemoderivados no país, e que entre o número total de casos acumulados de AIDS o percentual de casos em que o HIV foi adquirido por esta via não é grande (3\%), este crédito de quase $50 \%$ dos entrevistados parece refletir um conceito e uma insegurança da população em relação à qualidade de serviços de saúde pública prestados no país que merecia maior atenção por parte das autoridades sanitárias.

Apesar do conhecimento sobre AIDS ser alto, muitas DST são ainda desconhecidas, exceto a gonorréia, a sífilis e o cancro mole. Conhecer e buscar tratamento adequado para as DST é uma importante forma de proteção, já sua presença aumenta o risco de contrair o HIV (Dalabetta et al., 1996; Grosskurth et al., 1996). O maior conhecimento sobre as DST está associado com maior escolaridade tal como já foi encontrado pela PNDS-1996. Comparando-se os resultados deste estudo com a PNDS-1996 diferenças porcentuais grandes são observadas, embora as doenças mais conhecidas sejam coincidentes, tal diferença pode estar refletindo uma maior escolaridade da amostra deste estudo.

\section{Percepção de risco}

As questões sobre percepção de risco qualificam o conhecimento sobre a epidemia, possibilitando perceber as interpretações que uma dada informação sofre até a sua apropriação pelo indivíduo e sua transformação em comportamento ou prática. No início da epidemia de AIDS devido ao uso do conceito de "grupos de risco", houve uma exclusão dos homens heterossexuais da construção da idéia de risco. Além disso, verificava-se que alguns estereótipos, superpostos às identidades e comportamentos escapavam aos conceitos previamente definidos. Alguns autores mencionaram que a definição de identidade masculinidade passa por práticas sexuais. Assim, o "papel de ativo" na relação sexual, definiria a masculinidade, independente do sexo de quem seria o "passivo" (Parker, 1991). No que se refere à AIDS, a crença de que este era um problema de homossexuais, posteriormente de mulheres, fez com que os homens se sentissem protegidos da infecção, daí a importância de investigar a percepção de risco entre estes homens.

Do grupo que diz não ter risco ou ter risco pequeno, $78 \%$ faz esta afirmação a partir de uma avaliação da sua situação: $53 \%$ disseram ter só uma parceira e $25 \%$ usaram preservativo. Dos que disseram ter risco moderado, $26 \%$ atribuíram esta avaliação ao fato de vir a precisar de uma transfusão de sangue. Trinta e cin- 
co por cento reconheceram o risco mas não se protegem, ou porque não usaram preservativo (10\%), ou porque acharam que possivelmente tiveram relação com alguém contaminado sem saber (14\%), ou porque têm ou tiveram muitas parceiras (11\%).

Apesar de ser pequeno o porcentual de homens (5\%) que dizem explicitamente não ter risco porque confiam na parceira, chama atenção por ser esta uma atitude semelhante à de algumas mulheres, para quem a imunidade ao HIV é conferida pela presunção do comportamento do outro, mais do que por alguma atitude ativa no sentido da auto proteção (Guimarães, 1996). Além disso, pode-se considerar que $53 \%$ que referiram ter apenas uma parceira também utilizam o mesmo raciocínio para avaliar seus riscos.

Embora haja respostas como "não acredito que AIDS exista" ou "não convivo com quem tem $A I D S$ " e que, ainda, haja uma atitude "fatalista" por parte de alguns dos entrevistados que assume o risco moderado por não usar camisinha e ter muitas parceiras, estes conceitos distorcidos apareceram com uma freqüência baixa entre os entrevistados. A atitude fatalista facilmente se confunde com a negação do risco, como no primeiro exemplo. Esta atitude "fatalista”, eventualmente relacionada a estereótipos masculinos de invulnerabilidade ou destemor, foi mais freqüentemente encontrada entre os homens das classes D/E.

Embora apenas 3\% dos homens que referiram nenhum ou risco pequeno tenham dito que o motivo relacionava-se a não ter tido relações com homossexuais, isto mostra a permanência do conceito de "grupo de risco", embora numa proporção bastante pequena.

\section{Prevenção da AIDS}

Mais da metade dos informantes responderam que sexo seguro era sexo com uma parceira conhecida, sendo que este resultado reitera a idéia identificada anteriormente, de que muitos entrevistados referem-se ao comportamento do outro para garantir a sua proteção. Embora não tenha sido perguntado a definição de "parceira conhecida", sabe-se por estudos anteriores que esta aparece como uma definição, ora referida ao tempo de conhecimento, ora à intensidade da paixão ou mesmo ao círculo de amigos comuns, o que é bem precário, se considerarmos a questão da proteção ao HIV ( Barbosa, 1997).

\section{Uso do preservativo}

Os dados da PNDS-1996 (BEMFAM/Macro International, 1997) mostram um aumento do uso do preservativo quando utilizado como método anticoncepcional. No Estado de São Paulo este uso que era de 3,1\% em 1986 (Arruda et al., 1987) cresceu para 6,9\% em 1996. O seu uso para a prevenção da AIDS, tendo sido muito maior: $14,4 \%$ para mulheres e $30,9 \%$ para homens na última relação sexual (Badiani et al., 1997). O aumento do uso do preservativo masculino pode ser observado também em outras esferas. De acordo com a empresa Nielsen, especializada em pesquisa de mercado, o volume de vendas de preservativo quadruplicou entre 1991 a 1996. As vendas anuais do produto no Brasil, que eram de 50 milhões em 1991, subiram para 200 milhões por ano em 1996 (Family Health International, 1997).

Este estudo, entre homens da cidade de São Paulo, revela maior uso de preservativo nos homens jovens e nas relações extras maritais, nos homens de maior escolaridade e aqueles com maior percepção de risco. Vários autores estudando o uso do preservativo têm referido que a idade parece ser um importante fator preditivo deste uso, o que pode refletir o fato de que indivíduos jovens por estarem sendo melhor educados em relação às suas necessidades de proteção, podem ter atitudes mais positivas em relação ao uso de preservativo (Grimley et al., 1997). Filipe \& Newman (1998) estudando uma amostra de homens dos serviços de saúde na cidade de São Paulo apontaram para um alto nível de uso de preservativo com parceiros, mulheres e homens, independente do estado sorológico do indivíduo. Os autores enfatizaram maior uso nas relações extra maritais. Mesmo assim, é importante frisar que muitos homens estão expostos ao riscos, devido às múltiplas parcerias conjugada ao não uso do preservativo ou várias parceiras sucessivas sem uso do preservativo. Segundo Fisher (comunicação pessoal), as mensagens educativas de Saúde Pública são reinterpretadas em comportamento de risco. É o caso da mensagem que estimula as pessoas a diminuir o número de parceiros sexuais e elas se engajam em monogamia seriada, que significa um parceiro por vez, mas pode significar vários ao longo do tempo.

\section{Conclusões}

As campanhas educativas de prevenção da AIDS que estimulam o uso do preservativo masculino parecem estar sendo bem sucedidas, principal- 
mente entre homens mais jovens. Campanhas similares abordando os riscos e a necessidade de tratamento e controle das DST devem também ser estimuladas, especialmente para a população de menor escolaridade e portanto de maior vulnerabilidade. Urge a inclusão de homens heterossexuais no escopo dos programas de prevenção da AIDS, assim como a questão da monogamia seriada.

\section{Agradecimentos}

Este estudo foi financiado pela Fundação Ford a quem os autores agradecem pelo apoio financeiro. Os autores também agradecem às várias pessoas envolvidas neste trabalho e em especial ao Prof. Dr. Nicholas John Ford, da Exeter University, que foi consultor técnico do Projeto, à Prof. Dra. Joanne Mantell, do HIVCenter, Columbia University, por suas várias sugestões, à Prof. Dra.Cássia Maria Buchalla, da Faculdade de Saúde Pública da Universidade de São Paulo, ao Prof. Dr. Afonso Dinis Costa Passos e ao Prof. Dr. Uilho Antonio Gomes, do Departamento de Medicina Social da Faculdade de Medicina de Ribeirão Preto, Universidade de São Paulo pelas valiosas sugestões ao manuscrito.

\section{Referências}

ARRUDA, J. M.; RUTENBERG, N.; MORRIS, L. \& FERRAZ, E. A., 1987. Pesquisa Nacional sobre Saúde Materno Infantil e Planejamento Familiarl PNSMIPF, Brasil 1986. Rio de Janeiro: Sociedade Civil Bem-Estar Familiar no Brasil/Institute for Research Development.

BADIANI, R.; QUENTAL, I. \& SANTOS, E. M., 1997. DST/AIDS e a Pesquisa Nacional sobre Demografia e Saúde: Uma Análise de Conhecimento e Comportamentos de Vulnerabilização. Rio de Janeiro: Sociedade Civil Bem-Estar Familiar no Brasil/Demographic and Health Surveys.

BARBOSA, R. M., 1997. Negociação Sexual ou Sexo Negociado. Tese de Doutorado, Rio de Janeiro: Instituto de Medicina Social, Universidade do Estado do Rio de Janeiro.

BEMFAM (Sociedade Civil Bem-Estar Familiar no Brasil) / MACRO INTERNATIONAL, 1997. Pesquisa Nacional sobre Demografia e Saúde, Brasil 1996. Rio de Janeiro: BEMFAM/Macro International.

CAPMBELL, C. A., 1995. Male gender roles and sexuality: Implications for women's AIDS risk and prevention. Social Science and Medicine, 41:197-210.

CONFERÊNCIA INTERNACIONAL SOBRE POPULAÇÃO E DESENVOLVIMENTO, 1994. Relatório. Brasília: Comissão Nacional de População e Desenvolvimento/Fundo de População das Nações Unidas.
DALLABETTA, G.; LAGA, M. \& LAMPTEY, P., 1996. Control of Sexually Transmitted Diseases: A Handbook for the Design and Management of Programs. Arlington: AIDS Control and Prevention Project, Family Health International.

EDUARDO, O. C.; MARCHI, A.; SILVA, N. \& ALMEIDA, P. M., 1979. Preparação para um Novo Critério de Classificação Sócio-Econômica. São Paulo: Associação Brasileira de Pesquisa de Mercado.

FAMILY HEALTH INTERNATIONAL, 1997. AIDS Control and Prevention Program. Final Report for the AIDSCAP Program in Brazil Sept 92/97. Arlington: Family Health International.

FILIPE, E. M. V. \& NEWMAN, S. P., 1998. Influence of HIV positive status on sexual behavior among males. Revista Saúde Pública, 32:503-513.

FUNDAÇÃO SEADE (Fundação Sistema Estadual de Análise de Dados), 1994. Município de São Paulo. Projeção da População Residente. São Paulo: Fundação SEADE.

GIFFIN, K., 1998. Beyond empowerment: Heterosexualities and the prevention of AIDS. Social Science and Medicine, 46:151-156.

GOLDSTEIN, D. M., 1994. AIDS and women in Brazil: An emerging problem. Social Science and Medicine, 39:919-929.

GOMEZ, C. A. \& MARIN, B. V. O., 1996. Gender, culture 
and power: Barriers to HIV prevention strategies for women. Journal of Sex Research, 33:355-362.

GRIMLEY, D. M.; PROCHASKA, G. E. \& PROCHASKA, J. O., 1997. Condom use adoption and continuation: A transtheoretical approach. Health Education Research, 12:61-75.

GROSSKURTH, H.; MOSHA, F; TODD, J.; MWIJARUBI, E.; KLOKKE, A.; SENKORO, K.; MAYAUD, P.; CHANGALUCHA, J.; NICOLL, A.; KA-GINA, G.; NEWELL, J.; MUGEYE, K.; MABEY, D. \& HAYES, R., 1995. Impact of improved treatment of sexually transmitted diseases on HIV infection in rural Tanzania: Randomised controlled trial. Lancet, 346:530-536.

GUIMARÃES, C. D., 1994. Mulheres, sexualidade e AIDS: Um projeto de prevenção. In: Alternativas Escassas: Saúde, Sexualidade e Reprodução (A. O. Costa \& T. Amado, org.), pp. 249-281, São Paulo: Fundação Carlos Chagas/Editora 34.

GUIMARÃES, C. D., 1996. Mas eu conheço ele! Um método de prevenção do HIV/AIDS. In: Quebrando o Silêncio: Mulheres e AIDS no Brasil (R. Parker \& J. Galvão, org.) pp. 169-179, Rio de Janeiro: Associação Brasileira Interdisciplinar de AIDS/Editora Relume-Dumará/Instituto de Medicina Social, Universidade do Estado do Rio de Janeiro.

HEISE, L. L. \& ELIAS, C., 1995. Transforming AIDS Prevention to meet women's need: A focus on developing countries. Social Science and Medicine, 40: 931-934.

HUBERT, M.; BAJOS, N. \& SANDFORT, T., 1998. Sexual Behaviour and AIDS in Europe. London: UCL Press.

LAUMANN, E. O.; GAGNON, J. H.; MICHAEL, R. T. \& MICHAELS, S., 1994. The Social Organization of Sexuality: Sexual Practices in the United States. Chicago: University of Chicago Press.

LERIDON, H., 1994. Number, sex and type of partners. In: Sexual Behaviour and AIDS (A. Spira \& N. Bajos, ed.), pp. 104-112, Avebury: ACSF Group.
LOYOLA, M. A., 1994. AIDS e prevenção da AIDS no Rio de Janeiro. In: Aids e Sexualidade: O Ponto de Vista das Ciências Humanas (M. A. Loyola, org.), pp. 19-72, Rio de Janeiro: Editora Relume-Dumará/Instituto de Medicina Social, Universidade do Estado do Rio de Janeiro.

MS (Ministério da Saúde), 1999. Distribuição dos casos de AIDS segundo a categoria de exposição, período de diagnóstico e sexo, Brasil 1980-1999. Semana Epidemiológica 35 a 47. Boletim Epidemiológico - AIDS, 11:26.

MENESIA, E. O., 1999. Estudo Epidemiológico sobre a Sindrome da Imunodeficiência Adquirida (AIDS) no Município de Ribeirão Preto. Dissertação de Mestrado, Ribeirão Preto: Faculdade de Medicina de Ribeirão Preto, Universidade de São Paulo.

PARKER, R., 1991. Corpos, Prazeres e Paixões: A Cultura Sexual no Brasil Contemporâneo. São Paulo: Editora Best-Seller.

PRANDI, R., s.d. Código de Classificação de Trabalho. São Paulo: Universidade de São Paulo.

SCPR (Social and Community Planning Research), 1990. National Survey of Sexual Attitudes and Lifestyles. London: SCPR.

SPSS INCORPORATION, 1994. SPSS for Windows. Statistical Package for the Social Sciences. Release 6.1. Chicago: SPSS Inc.

VILLELA, W. V., 1996. Refletindo sobre a negociação sexual como estratégia de prevenção da AIDS entre mulheres. In: Quebrando o Silêncio: Mulheres e AIDS no Brasil (R. Parker \& J. Galvão, org.), pp. 181-189, Rio de Janeiro: Associação Brasileira Interdisciplinar de AIDS/Editora Relume-Dumará/ Instituto de Medicina Social, Universidade do Estado do Rio de Janeiro.

VILLELA, W., 1997. Homens que Fazem Sexo com Mulheres: Propostas e Pistas para o Trabalho. São Paulo: Núcleo de Estudos e Prevenção da AIDS, Universidade de São Paulo. 